\title{
ANALYSIS OF MFCC FEATURES FOR EEG SIGNAL CLASSIFICATION
}

\author{
Gnana Rajesh D, \\ Department of Information Technology, \\ Al Musanna College of Technology, \\ Sultanate of Oman \\ rajesh@act.edu.om
}

\begin{abstract}
In this paper, an experimental evaluation of Mel-Frequency Cepstral Coefficients (MFCCs) for use in Electroencephalogram (EEG) signal classification is presented. The MFCC features are tested using CHB-MIT Scalp EEG Database. The objective is to classify the given EEG signal into normal or abnormal that is based on the MFCC representation of EEG signal. Initially, the QRS complex waves are detected using Pan Tompkins algorithm, and then the MFCC features are extracted. The performance of MFCC feature representation is analyzed in the context of an Artificial Neural Network (ANN) classification system in terms of sensitivity and specificity. The performance of EEG classification approach depends on the number of MFCC components used for the classification. When compared with 15 and 35 MFCC components, 25 MFCC components gives better result in terms of sensitivity (98\%) and specificity (96\%).
\end{abstract}

Keywords: Electroencephalogram, Pan Tompkins Algorithm, MFCC Features, ANN Classifier.

\section{INTRODUCTION}

The abnormalities in the human brain are analyzed with the help of EEG recordings. The accuracy of mutual information based EEG signals classification system is increased in [1] by a hybrid approach. In a two-class mutual information recognition system, a Least Square Support Vector Machine (LS-SVM) classifier is employed for EEG classification using cross-correlation based feature. In order to verify the classifier performance, the LS-SVM classifier is replaced by a logistic classifier based on kernel logistic regression and conventional regression separately.

A method of EMD-chaos based system to discriminate EEG signals concerning healthy persons and epileptic patients is discussed in [2]. It is very useful during seizure attacks and seizure-free intervals. Initially, EEG is decomposed empirically during the intrinsic mode functions. An EEG signal classification system discussed in [3] makes use of the number of subject dependent parameters such as best common spatial pattern channels and the best frequency range of the channels of the system. Using these parameters the energy features are extracted and classified using the LS-SVM classifier as a back end classification.

In [4], EEG signals are decomposed to five brain rhythms after the preprocessing and windowing procedure using the discrete wavelet transform. Lorenz inconsistent and consistent features are extracted using the singular Lorenz measures method. Then, a hybrid form of K-Nearest Neighbor (KNN) and Scatter Search (SS) classification algorithms are used for the classification process.

A method known as centroid based binary tree structured SVM is discussed in [5]. The binary of the SVM models as well as the similarities of the 
class labels distances from the respective root level centroids are found for EEG classification. The classification of multi-class self-paced motor imagery is achieved by least-square LS-SVM in [6]. It is used to classify the temporal features while tuning the hyper parameters automatically.

In order to detect epilepsy, an enhanced classifier with modified fuzzy clustering algorithm is used in [7]. It makes use of the Bayesian theory. The selection of learning parameter of the clustering membership is chosen based on the randomly selected clustering centre degree.

EEG signals are filtered by regularized spatiotemporal approach for the classification in [8] by using a single trial EEG. The system consists of two steps such as; feature extraction step, an 12-regularized scheme is used for supervised spatiotemporal filtering and sparse Fisher linear discriminant analysis is used in EEG classification where the optimization is achieved using a convex optimization approach.

The raw EEG signals are preprocessed by surface Laplacian filtering [9] and is decomposed by using the wavelet transform into five various EEG frequency types like alpha, beta, gamma, delta, and theta. Then the wavelets like $\mathrm{db} 4, \mathrm{db} 8$, sym 8 and coif5, are used for extracting features and classified by KNN classifier. The importance of feature and channel selection for the classification of EEG signal is discussed in [10]. The selection process is done by searching the feature/channel space using a genetic algorithm and calculating the importance of a linear SVM classifier.

An ensemble synchronization measure in [11] is used for the classification with the help of EEG signal pairs. Using ensemble synchronization scheme and latency response of the EEG recordings are used as features. Then, logistic regression is used to classify each single trial of EEG. Dempster-Shafer theory based EEG classification with KNN classifier is discussed in [12]. The autoregressive EEG models and wavelet decomposition are used for extracting features. KNN scheme is used for EEG classification.

\section{METHODS AND MATERIALS}

Figure 1 shows EEG signal classification system using MFCC features and ANN classifier. At first, the EEG signals are preprocessed to divide the whole signals into small segments (QRS peaks) by using Pan Tompkins algorithm [15]. Features are extracted from the small segments using MFCC, and these MFCC features are analyzed using ANN for abnormality detection.

\section{A. Pan Tompkins Algorithm}

The system for EEG signal classification uses the QRS detection algorithm in [15] to extract the QRS peaks in the given signal. The detection of QRS is based on the width, slope and amplitude. It is a complex process. Specifically designed band pass filter is designed to eliminate the interferences caused by electrode motion, baseline wander, power line interference and high frequencies $\mathrm{T}$ waves similar to QRS waves.

The QRS detection in done by using the following three phases. In the learning phase, the thresholds are detected with the help of 2 seconds of EEG signal. The next learning phase, the limit and average RR-interval values are identified with the help of two heart beats. At last, the pulse for QRS complex is recognized in the subsequent recognition stage. 


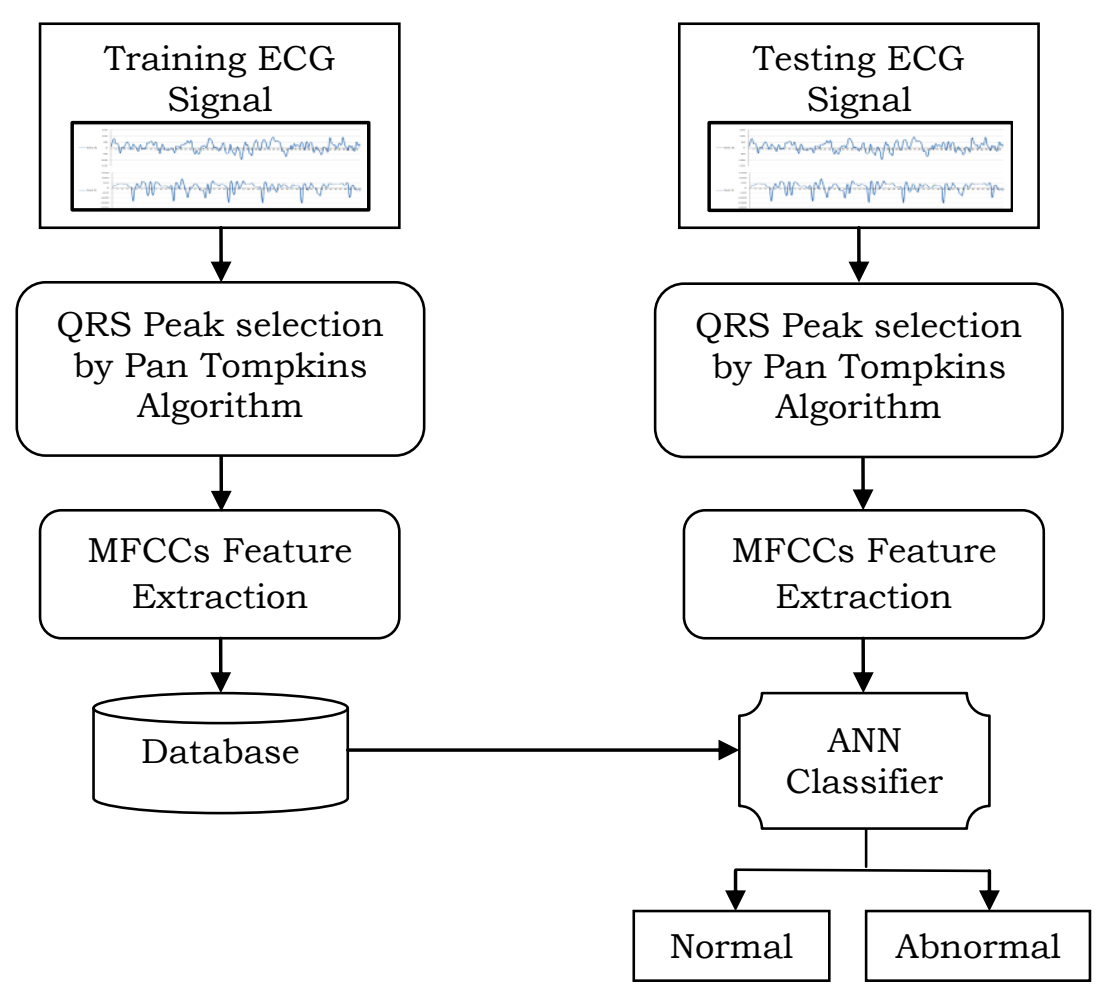

Fig. 1 Block diagram of EEG signal Classification system

\section{B. MFCCs Feature Extraction}

MFCCs are a crudely separate source and filter [13]. Because of its nonlinear frequency scale, de-correlated nature, and robustness to noise, this representation is widely used in signal processing. Usually, Discrete Fourier transform (DFT) is used to compute MFCC parameters of the windowed speech signal using Mel-binned filters. $X[k]$ is the DFT of one frame of the signal, $x[n]$ :

$$
X[K]=\sum_{n=0}^{N-1} x[n] e^{\frac{-j 2 \pi n k}{N}} 0 \leq k<N
$$

Then, a filter bank with triangular filters spaced along a Mel-warped frequency scale is constructed, as shown in Figure 2. This filter bank is applied to the DFT coefficients. The outputs of the filter bank are then subjected to logarithmic compression. This logarithmic operation is a homomorphic transformation, a transform that converts a convolution into a sum.

Finally, MFCCs are computed using Discrete Cosine Transform (DCT) from the above log Mel-binned filter bank coefficients ( $m 1)$, as shown below [14]:

$$
M F C C_{i}=\sqrt{\frac{2}{N}} \sum_{j=1}^{N} m_{j} \cos \left(\frac{\pi i}{N}(j-0.5)\right)
$$

The higher order Cepstral coefficients are significantly smaller in magnitude than the lower ones. Hence, in order to normalize the magnitudes of 
these coefficients, Cepstral liftering is used. This is done by using the following formula [14]:

$$
c_{n}^{1}=\left(1+\frac{L}{2} \sin \frac{\pi n}{L}\right) c_{n}
$$

where $L$ is the lifting parameter $(L=2(N-1)), c_{n}$ and $c_{n}^{1}$ are Cepstral coefficients of before and after lifting.

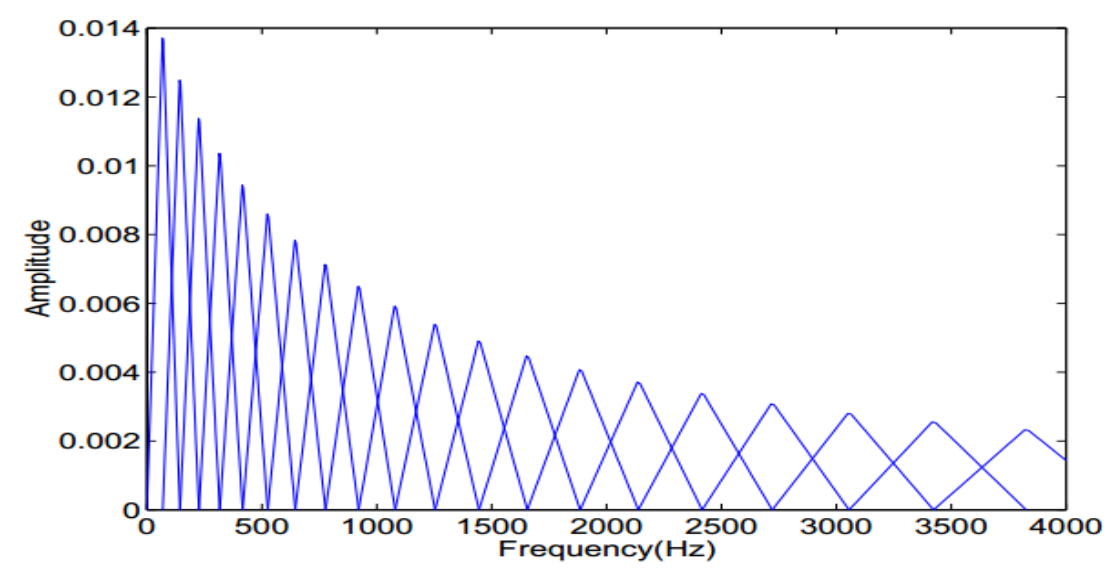

Fig. 2 Mel-warp triangular filters

\section{Classification}

ANN algorithm is used for EEG signal classification which is a supervised neural network. It uses back propagation algorithm for effective classification. At each node, a differential transfer function is included. One of the advantages of using back propagation is that it can generate complex decision boundaries and can modify the weights of each neuron at iteration. Figure 3 shows the architecture of back propagation network. It consists of three types of layers for processing. The input layer consists of $\mathrm{N}$ number of neurons which depends on the length of given input features. For 25-MFCC components, the input layer consists of 25 neurons. In general, the number of hidden layers is $2 \mathrm{~N}+1$. The number of the output layer is one as it simply classifies the given data into normal or abnormal.

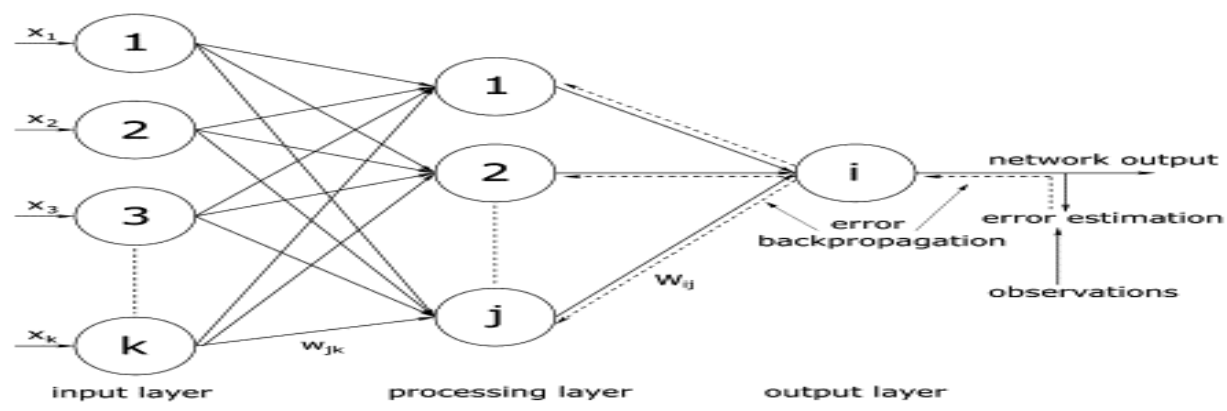

Fig. 3 Architecture of back propagation neural network 


\section{RESULTS AND DISCUSSION}

The EEG classification system is tested with the CHB-MIT Scalp EEG Database [15-16]. A total of 100 EEG signals (50 normal and 50 abnormal) are randomly selected from the database. The system is tested with ten runs of simulation. At each run, only five signals from both normal and abnormal category are randomly chosen for testing. 25 and 35 MFCC coefficients are analyzed. Figure 4 shows the performance of EEG signal classification system using MFCC features and ANN classifier in terms of accuracy, sensitivity, and specificity.

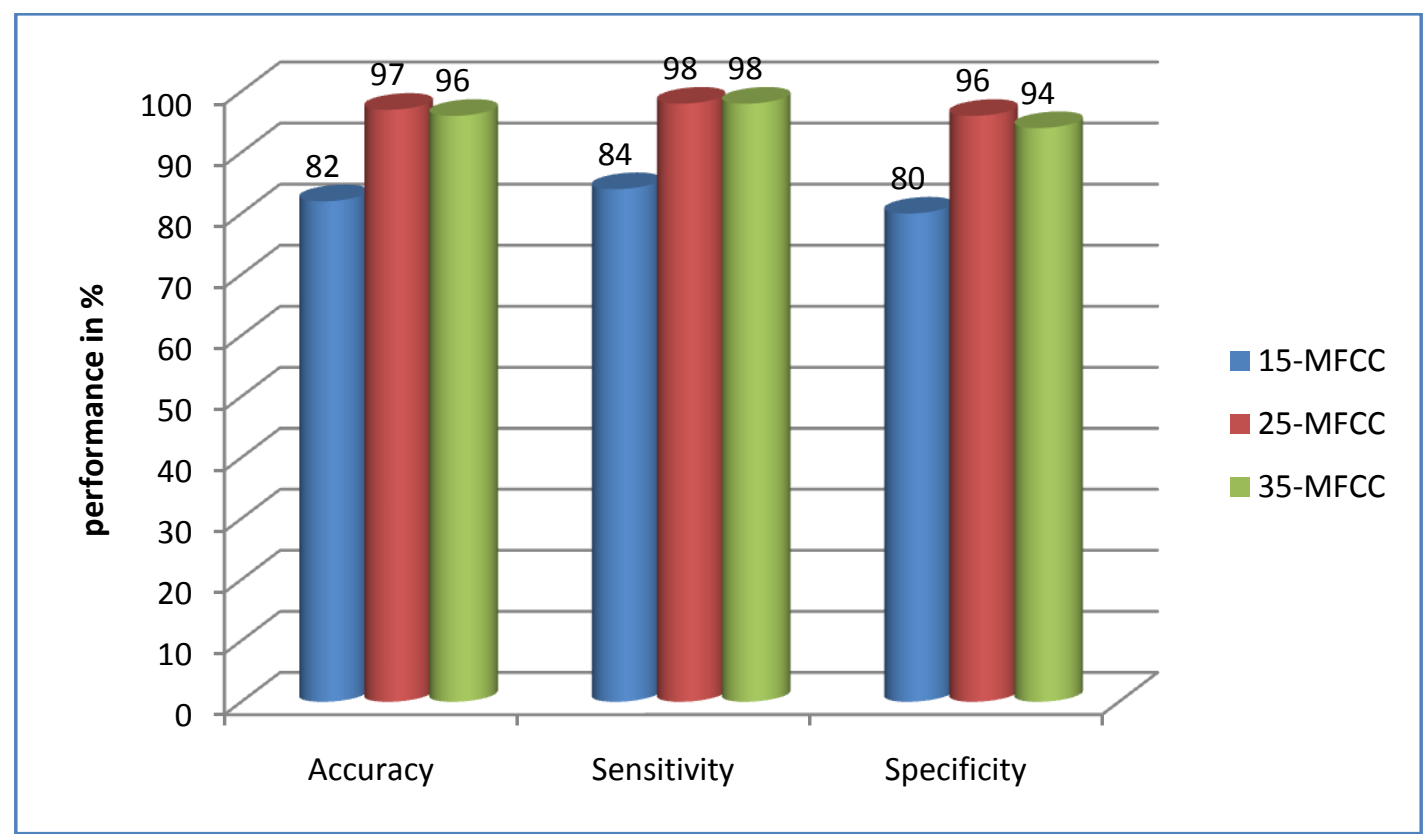

Fig. 4 Performance of EEG signal classification

It is observed from the results that $25 \mathrm{MFCC}$ components are enough to provide a maximum accuracy of $97 \%$ with $98 \%$ sensitivity and $96 \%$ specificity. While increasing the MFCC components from 25 to 35, the specificity of the system is reduced from $98 \%$ to $94 \%$ that affects the overall system accuracy.

\section{CONCLUSION}

In this paper, the abnormal activities of the human brain are detected by using MFCC and ANN. MFCC components capture the characteristics of abnormal pulses in the QRS complex automatically. In ANN, the error contribution of each neuron is computed using back propagation algorithm. Three different setups are tested for getting better accuracy with 15-MFCCs, 25-MFCCs, and 35-MFCCs. The $25-M F C C$ s setup shows the best performance than other setups with $97 \%$ accuracy with $98 \%$ sensitivity and $96 \%$ specificity. In future research, feature selection could be used to reduce the feature dimension by selecting best MFCCs. 


\section{REFERENCES}

[1]. S. Siuly, and Y. Li, "Improving the separability of motor imagery EEG signals using a cross correlation-based least square support vector machine for brain-computer interface", IEEE Transactions on Neural Systems and Rehabilitation Engineering, Vol. 20, No. 4, 2012, pp. 526-538.

[2]. S.S. Alam, and S. TarekShahriar, "EEG signal discrimination using nonlinear dynamics in the EMD domain", International Journal of Computer and Electrical Engineering, Vol. 4, No. 3, 2012, pp.326-330.

[3]. M.E. Abdel-Hadi, R.A. El-Khoribi, M.I. Shoman, and M.M. Refaey, "Classification of motor imagery tasks with LS-SVM in EEG-based selfpaced $\mathrm{BCI}$ ", IEEE $5^{\text {th }}$ International Conference on Digital Information Processing and Communications, 2015, pp. 244-249.

[4]. M. Behnam, and H. Pourghassem, "Singular Lorenz Measures Method for seizure detection using KNN-Scatter Search optimization algorithm", IEEE Signal Processing and Intelligent Systems Conference, pp. 67-72, 2015.

[5]. A. Govada, B. Gauri, and S.K. Sahay, "Centroid based Binary Tree Structured SVM for multi classification". IEEE International Conference on Advances in Computing, Communications and Informatics, 2015, pp. 258-262.

[6]. M. Hamedi, S.H. Salleh, C.M. Ting, A.M. Noor, and I.M. Rezazadeh, "Multiclass self-paced motor imagery temporal features classification using least-square support vector machine", IEEE 19 Electrical Stimulation Society Annual Conference, 2014, pp. 1-5.

[7]. P. Jaiswal, and R. Koushal, "EEG signal classification using Modified Fuzzy Clustering algorithm", International Journal of Computer Science And Information Technologies, Vol. 6, No. 3, 2015, pp. 2031-2034.

[8]. F. Qi, Y. Li, and W. Wu, "RSTFC: A novel algorithm for spatio-temporal filtering and classification of single-trial EEG", IEEE transactions on neural networks and learning systems, Vol. 26, No. 12, 2015, pp. 30703082.

[9]. M. Murugappan, "Human emotion classification using wavelet transform and KNN", IEEE International conference on Pattern analysis and intelligent robotics, 2011, pp. 148-153.

[10]. A. Al-Ani, and A. Al-Sukker, "Effect of feature and channel selection on EEG classification", IEEE $28^{\text {th }}$ Annual International Conference on Engineering in Medicine and Biology Society, 2006, pp. 2171-2174.

[11]. P.D. Prasad, H.N. Halahalli, J.P. John, and K.K. Majumdar, "Single-trial EEG classification using logistic regression based on ensemble synchronization", IEEE journal of biomedical and health informatics, Vol. 18, No. 3, 2014, pp. 1074-1080.

[12]. A. Yazdani, T. Ebrahimi, and U. Hoffmann, "Classification of EEG signals using Dempster Shafer theory and a k-nearest neighbor classifier", IEEE $4^{\text {th }}$ International IEEE/EMBS Conference on Neural Engineering, 2009, pp. 327-330.

[13]. S.B. Davis, and P. Mermelstein, "Comparison of parameteric representations for monosyllabic word recognition in continuously spoken sentences", IEEE Trans. Acoustics, Speech, Signal Processing. Vol. 28, No. 4, 1980, pp. 357-366.

[14]. S. Young, J. Odell, D. Ollason, V. Valtchev, and P. Woodland, "The HTK Book", Version 2.1, Cambridge University, Entropic Cambridge Research Laboratory, UK, 1997.

[15]. J. Pan, and W.J. Tompkins, "A real-time QRS detection algorithm", IEEE transactions on biomedical engineering", Vol. 3, 1985, pp. 230-236. 
[16]. A. Shoeb, "Application of Machine Learning to Epileptic Seizure Onset Detection and Treatment", PhD Thesis, Massachusetts Institute of Technology, 2009.

[17]. A.L. Goldberger, L.A.N. Amaral, L. Glass, J.M. Hausdorff, P.C.H. Ivanov, R.G. Mark, J.E. Mietus, G.B. Moody, C.K. Peng, and H.E. Stanley, "PhysioBank, PhysioToolkit, and PhysioNet: Components of a New Research Resource for Complex Physiologic Signals", Circulation, Vol. 101, No. 23, pp. e215-e220. 